\title{
Les objets grammaticaux dans les pratiques en classe de français, langue première et étrangère
}

\author{
Sandra Canelas-Trevisi \\ LIDILEM - Université Stendhal Grenoble 3 \\ GRAFE - Université de Genève \\ $\underline{\text { Sandra.Trevisi@u-grenoble3.fr }}$
}

\section{Introduction}

La présente intervention s'attache à développer un thème de recherche, les pratiques en classe autour des objets grammaticaux enseignés, et à le mettre en perspective par rapport à la sectorisation de la didactique du français. Il est abordé selon deux points de vue : les savoirs de référence d'une part, la séquentialité des actions verbales et non verbales repérables en situation, d'autre part.

Le thème choisi sollicite une pluralité de références. De l'analyse du travail à celle de l'interaction, des théories de l'action à celles de l'apprentissage et de l'acquisition sans oublier, bien entendu, les théories linguistiques qui ont influencé l'enseignement à travers l'histoire. Un couple de questions permet de délimiter notre problématique à l'intérieur de cet espace très large : quel modèle de la langue faut-il se donner pour l'enseigner d'une part et comment les objets transmis déterminent les actions inhérentes à leur enseignement et à leur apprentissage d'autre part.

La théorie de la transposition apporte la première légitimation du point de vue adopté : les objets mis en circulation dans les pratiques en classe sont inévitablement différents de ceux qu'une institution et plus largement la société désignent comme contenu de l'enseignement et comme repères pour l'évaluation des apprentissages. Ce sont des objets institutionnels analysables, ils prennent forme dans le système didactique et ils sont surdéterminés par les contraintes de son fonctionnement.

L'étude de ces objets et des conditions de leur enseignement est intégrée dans la recherche et dans la formation initiale des enseignants. Est-ce que ceux d'entre eux qui se forment pour l'enseignement à des publics francophones sont confrontés aux mêmes analyses critiques et aux mêmes cadres théoriques que leurs homologues engagés dans des formations de français pour non francophones? Est-il justifié de parler de deux didactiques de la grammaire?

Indice anecdotique mais néanmoins significatif de la fragilité de leurs frontières respectives, les dénominations mêmes des deux didactiques du français sont aujourd'hui sujettes à discussion. Que signifie langue maternelle dans un environnement plurilingue et multiculturel ? Ne faudrait-il pas plutôt parler de langue socialement première, définie comme langue de la communauté linguistique majoritaire, langue de la société civile et de l'Etat, adoptée par les immigrants, et par conséquent langue de la scolarité obligatoire (Chartrand, 2003) ? De même, peut-on faire l'économie d'une réflexion approfondie sur la distinction entre langue étrangère et langue seconde dans un environnement où la présence de deux ou plusieurs langues nationales est reconnue à des degrés divers dans les institutions éducatives ?

Nous adoptons les dénominations de Didactique du Français Langue Première (DFLP) et Didactique du Français Langue Etrangère (DFLE) pour désigner deux entités institutionnelles, sans pour autant leur attribuer une valeur définitive, mais considérant plutôt qu'elles sont le résultat de décisions prises par une communauté de chercheurs à un moment donné et, à ce titre, révélatrices de positions institutionnelles et épistémologiques. La question qui nous préoccupe est la suivante : comment abordent-elles les objets grammaticaux? 


\section{Un appareil grammatical en FLP}

\subsection{Des objets multidimensionnels}

En admettant que tout objet apprêté pour être enseigné est le produit d'un processus de transformation, le didacticien délimite un espace de problèmes à théoriser. La théorie de la transposition didactique lui apporte des outils pour le faire. Nous avons testé les analyseurs qu'elle propose et nous avons pu en évaluer la pertinence et les limites (Canelas-Trevisi, 1997). Certains ont voulu voir dans la théorie de la transposition une démarche visant exclusivement à illustrer les transformations du savoir savant intégré dans l'enseignement. Ils s'y sont alors opposés revendiquant le principe que l'intégration des savoirs dans le contenu de l'enseignement présuppose des formes de recomposition et des reconfigurations (Vargas, 2004). Nous considérons que cette objection ne met pas radicalement en question les contraintes qui caractérisent le processus de la transposition. A partir d'une théorie des textes et des discours (Bronckart, 1996), celles-ci peuvent en effet être considérées comme des déterminants du genre ou plutôt des genres de textes produits et utilisés dans les pratiques d'enseignement. Elles ne suffisent pas à les décrire, et de notre point de vue, la théorie ne le prétend pas.

Dans la discipline français, l'analyse critique des savoirs ${ }^{1}$ apprêtés pour l'enseignement prend en compte tous les composants explicites et implicites des objets à enseigner, notamment les rôles respectifs des dimensions susceptibles de les définir. Les objets d'enseignement du français, du fait de la concrétion propre au langage, au sens de Milner, s'intègrent nécessairement dans une perspective qu'on pourrait appeler « dimensionnelle ». Lorsqu'un objet de savoir est transposé pour devenir un objet à enseigner, il est extrait du continuum qui lui donne sens dans une pratique particulière et il s'intègre nécessairement dans l'une des dimensions du langage que l'école reconnaît. Celles-ci constituent dès lors des préconstruits dans lesquels les objets transposés vont s'inscrire. Le processus de la transposition rencontre ainsi inévitablement l'intrication des dimensions du savoir de l'enseignement institutionnel.

La multidimensionnalité des objets enseignés a été identifiée à l'aide d'oppositions diverses selon les époques. Les mouvements de rénovation de l'enseignement du français des années 1970 ont essayé d'articuler libération de la parole et structuration de la langue, aujourd'hui la didactique du français s'interroge sur les relations entre grammaires de la phrase, du texte et du discours et tente de concilier les implications socio-culturelles que ces domaines comportent. En FLE, après avoir admis implicitement que toute hétérogénéité pouvait se fondre dans l'approche communicative dominante, la recherche est aux prises avec les problèmes de l'explicitation et de l'apprentissage des formes linguistiques, dans l'interaction verbale d'une part, dans la construction du sens au cours des activités de classe sur les objets grammaticaux d'autre part.

Les pratiques sociales de référence des objets à enseigner sont souvent mentionnées dans un but de valorisation et de légitimation des contenus enseignés. Parallèlement, la délimitation et la reconstruction des objets sont annoncées dans l'institution, mais leurs conséquences pour les pratiques en classe ne sont pas suffisamment étudiées. La problématique des modèles didactiques et des technologies d'intervention qui les accompagnent est aujourd'hui au cœur de la didactique. La présente contribution se situe dans cette problématique en posant deux questions: quelles sont les caractéristiques des pratiques en classe autour des objets grammaticaux, objets multidimensionnels, cumulant des références à des savoirs hétérogènes ? Comment concevoir en parallèle le modèle didactique des objets grammaticaux à enseigner et l'ingénierie didactique pour la mobilisation de ces objets grammaticaux en situation de classe?

\subsection{Un système d'objets grammaticaux à reconfigurer ?}

Dans le domaine du français langue première, nous discuterons en particulier l'appareil grammatical en vigueur en Suisse romande, dans l'école secondaire inférieure. Ses grandes lignes remontent à la rénovation de l'enseignement du français de la fin des années 1970 (Besson, Genoud, Lipp \& Nussbaum., 1979). Aujourd'hui, pour le modifier, il ne s'agit plus, de se référer à un modèle théorique dominant, mais 
de se demander lesquelles des théories disponibles sont susceptibles de fournir des éléments pertinents pour les projets didactiques d'intervention.

Pour apporter des éléments de réponse à cette question, l'analyse des documents pédagogiques en vigueur dans l'institution nous fournit un premier éclairage. Leur construction s'appuie sur le savoir de référence dominant dans les années 1970, 1980 : la grammaire générative, notamment sa première version. La mise en texte des objets grammaticaux destinés à l'enseignement révèle les traces de ce savoir de référence, transposé et reconstruit ainsi que de son articulation avec des savoirs de la tradition scolaire d'une part, des savoirs issues de théories totalement éloignés, comme la théorie de l'énonciation et celle des actes de parole, d'autre part.

Par delà cette pluralité d'apports, une analyse fine des documents en tant que textes montre que la cohérence de l'appareil d'analyse se fonde sur l'adhésion implicite aux notions de compétence et de locuteur idéal. Nous avons montré ailleurs (Canelas-Trevisi, sous presse) que les idéalités chomskyenne jouent un rôle essentiel. Elles apportent aux auteurs de l'ouvrage fondateur de la rénovation de l'enseignement la justification pour mettre en place une tentative originale : fondre les dimensions de l'expression de soi et de la production/compréhension orale et écrite, traditionnellement retenues dans l'école, avec la dimension de la prise de conscience de la langue. Les manipulations, issues des procédures syntaxiques de la théorie, constituent l'ingénierie didactique censée justifier la signification et la validité des objets grammaticaux. La construction de l'appareil d'analyse initial s'appuie sur l'hypothèse forte que la prise de conscience du fonctionnement de la langue, basée notamment sur les manipulations, permettrait d'organiser tous les autres aspects de l'enseignement. Cette hypothèse est très vite nuancée, dans les documents mêmes de l'institution destinés aux enseignants

Une des questions centrales qui se pose est celle du rôle à attribuer au modèle de la phrase de base et aux manipulations syntaxiques. Dans l'ouvrage De la phrase aux énoncés: grammaire scolaire et descriptions linguistiques sous la direction de M.-J. Béguelin (2000) sont présentés les résultats d'une réflexion collective sur l'enseignement de la grammaire menée pendant plusieurs années (Rapport intermédiaire du Groupe Bailly, Neuchâtel, IRDP, 1988) : «Commandé par la Commission romande des moyens d'enseignement (COROME) ce livre constitue à la fois un prolongement de la rénovation, une façon de faire le point et, sous quelques aspects, une tentative de dépassement » (Introduction, p. 9).

Le titre du chapitre 3, La phrase insaisissable, reflète la discussion sur l'inadéquation des critères de définition de la phrase hérités de la tradition grammaticale. Dans le chapitre 6 sont exposées les grandes lignes de l'appareil d'analyse de l'enseignement. En conclusion sont évoqués les aspects positifs et ceux qui demanderaient à être amendés. Parmi les premiers, sont mentionnées les manipulations. Parmi les inconvénients de l'enseignement actuel sont soulignés : la faiblesse des manipulations d'effacement et de permutation pour l'identification des compléments, notamment pour la distinction entre le complément de verbe et le complément de phrase ; le statut accordé à la phrase modèle, la Phrase $\mathrm{P}$, et le principe que toute phrase non canonique est considérée comme le produit d'une ou de plusieurs transformations effectuées sur la phrase de base sous-jacente.

Si l'on trouve intérêt, dans l'enseignement grammatical, à user d'un schéma phrastique abstrait, servant de point de référence pour analyser la variété structurelle des énoncés, il faudrait cependant trouver les moyens de dénier à ce schéma toute priorité d'ordre logique ou d'ordre normatif. (p. 132).

Sur quelle base réorganiser l'appareil d'analyse et surtout comment transformer l'ingénierie des manipulations? Il ne s'agit pas pour nous d'apporter une réponse tranchée, mais de rechercher des éléments pour réaliser un état des lieux préalable à toute décision d'intervention. A défaut de ce type d'investigation, une telle décision procédera inévitablement de l'adhésion à l'un ou l'autre des savoirs disponibles, sur la base d'hypothèses difficiles à formuler et encore plus à vérifier. L'analyse critique des pratiques de classe constitue, nous semble-t-il, un élément essentiel de l'état des lieux. 


\subsection{L'analyse critique des données de classe}

\subsubsection{Des séquences d'actions verbales et non verbales}

Parmi les recherches sur les pratiques en classe, le modèle de la situation didactique conçu par Brousseau s'est avéré très fécond. Il s'agit néanmoins de modéliser les phénomènes qui se produisent au cours de la situation d'enseignement/apprentissage d'un objet particulier dans un dispositif d'ingénierie. Par conséquent, les situations ordinaires observables dans les classes ne peuvent être appréhendées que très partiellement à l'aide de ce modèle (Schubauer-Leoni, M.-L., Leutenegger, F., Ligozat, F. \& Flückiger, A., 2007).

Ayant constaté lors d'analyses précédentes que le modèle de Brousseau laissait dans l'ombre des aspects à notre avis significatifs des conduites observées, nous avons adopté une démarche d'analyse qualitative qui aboutit à la reconstruction théorique des données, conjuguant les aspects inductif et déductif. De manière schématique, nous considérons les faits observables dans une classe comme une succession d'actions verbales et non verbales autour d'un objet institutionnel, enseigné et censé être appris. Les documents pédagogiques de l'institution fournissent des indices pour identifier les contours de l'objet enseigné. Nous considérons toutefois que la forme et le sens de l'objet observé se définissent en situation. Pour les cerner, nous nous appuyons sur la notion d'action telle qu'elle est définie par Jean-Paul Bronckart (2004) et ses collaborateurs, dans le cadre de l'interactionnisme socio-discursif ${ }^{2}$.

Notre objectif est de cerner ce que font les enseignants et les élèves lorsqu'ils interagissent avec des objets grammaticaux, sans préjuger de la conformité des actions et de la parole des uns et des autres avec les notions et les techniques préconisées. C'est pourquoi nous avons alors utilisé le terme générique d'intervention pour désigner toutes les formes d'action ayant trait aux données de langue (extraits de textes, phrases canoniques, groupes, mots, morphèmes) incluant l'objet enseigné. Notre questionnement porte sur le sens de ces interventions, que nous interprétons en établissant des relations entre forme d'intervention, éléments de l'objet et dimension du langage. Seulement après avoir interprété le sens de l'intervention en contexte, on peut décider si celle-ci peut être mise en relation avec une technique spécifique d'investigation. Rappelons que les manipulations se veulent une transposition des procédures codifiées dans la recherche en syntaxe. Notre démarche s'appuie ainsi sur des traces langagières, mais également sur l'interprétation des actions comme manifestations possibles des faits didactiques.

Parmi les objets grammaticaux inscrits dans les programmes de l'enseignement secondaire en Suisse romande, nous avons retenu la subordonnée relative parce qu'il s'agit d'un objet susceptible de révéler la conception des manipulations dans l'épistémologie de l'enseignant et le sens des manipulations dans l'action en classe. Ancrées dans un savoir historiquement identifiable, les manipulations sont considérées comme obsolètes par certains, comme trop contraignantes et au-dessus des capacités des élèves par d'autres. Parallèlement, il est très largement admis que tout travail en grammaire se fonde sur des procédures. Elles sont nécessaires pour dépasser une approche visant uniquement l'identification des bonnes formes à retenir. Le sens des procédures en situation constitue dès lors un indicateur important des conditions du travail en grammaire dans le contexte institutionnel. Aussi, son analyse critique peutelle s'avérer utile pour planifier les modifications à introduire dans l'appareil grammatical en vigueur, qui sont actuellement à l'étude.

\subsubsection{Eléments de méthodologie}

Nous nous bornons ici à commenter les analyses de données recueillies dans deux classes, ci-après AM et BS, de l'école secondaire inférieure à Genève. Le recueil a été effectué dans le cadre d'un projet de recherche sur La construction de l'objet enseigné, mené par le Groupe d'Analyse du Français Enseigné (GRAFE), auquel nous avons collaboré (ouvrage en préparation sous la direction de B. Schneuwly et J. Dolz). Le contrat établi avec les enseignants comportait la mise en œuvre d'une séquence d'enseignement sur la subordonnée relative, objet grammatical inscrit dans les programmes. Chaque enseignant s'est engagé à organiser aussi bien le contenu que son déroulement temporel selon son propre 
programme. Un chercheur a observé et filmé les séquences. Aucune autre contrainte n'a été imposée aux enseignants.

La séquence $\mathrm{AM}$ est constituée de trois leçons, respectivement de 44, 43 et 41 minutes, enregistrées les 16, 17 et 23 janvier; la séquence BS de quatre leçons, respectivement de 40, 30, 42 et 43 minutes, enregistrées les 11 et 25 février de la même année 2003. L'analyse s'applique à un corpus textuel comportant :

- la transcription des interactions ;

- $\quad$ les documents mis en circulation dans la classe (exercices, textes);

- $\quad$ les éléments notés au tableau ;

- les notes du chercheur en cours d'observation.

Pour réduire les données afin de les appréhender et de les comparer, nous avons procédé en deux étapes. A partir de la lecture des transcriptions, nous avons repéré les lexèmes et les syntagmes verbaux et nominaux désignant l'objet et les interventions sur l'objet, ce denier terme étant à interpréter au sens générique précisé ci-dessus. Les résultats de ce premier balayage nous ont permis d'établir un tableau synoptique. Ensuite, sont repérées les déclencheurs d'avancement du temps didactique, susceptibles de marquer le début d'un enchaînement, caractérisé par le maintien d'une même finalité ${ }^{3}$. Les travaux sur la temporalité didactique (Sensevy, 1998) ont mis en évidence des phénomènes d'avancement du temps didactique, déclenchés par des indicateurs langagiers et/ou praxéologiques : les consignes de l'enseignant, la mise en circulation dans l'environnement d'un matériel nouveau (exemples, outils métalangagiers, schémas, exercices, données de langue diverses...). Nous avons considéré que ces déclencheurs marquent l'émergence d'une nouvelle finalité, dont le sens et la forme se définissent en situation. Ainsi, les indicateurs langagiers et praxéologiques ainsi que la finalité partagée constituent-ils des indices nous autorisant à réunir actions et objets dans une même unité d'analyse, que nous avons appelée enchaînement praxéologique et textuel. D'abord situés par rapport au déroulement temporel de la séquence, les enchaînements sont ensuite soumis à une analyse détaillée dont le but est de parvenir à interpréter les interventions sur les données de langue.

\subsubsection{Quelques résultats}

Les deux études de cas discutés ici montrent qu'en fin de scolarité obligatoire ${ }^{4}$ l'objet subordonnée relative est enseigné selon les principes de l'appareil institutionnel d'analyse et que la classe s'engage en apparence assez facilement dans les interventions sur les données de langue. Quel est le sens de ces interventions ? S'agit-il de produire des phénomènes pour tester une hypothèse ou pour mettre à l'épreuve une intuition?

Dans la séquence AM (pour une analyse détaillée voir Canelas-Trevisi sous presse), nous nous bornons ici à commenter un extrait de la dernière partie du troisième enchaînement, qui nous semble particulièrement révélateur des caractéristiques de l'objet enseigné et de sa mise en œuvre (les trois premiers enchaînements présentent des aspects récurrents: l'enseignant invite les élèves à agir en manipulant des exemples et leur demande ensuite de réfléchir sur ce qu'ils ont fait).

On sait que l'objet est élémentarisé pour être enseigné et qu'il est toujours à son tour élément d'un ensemble. L'objet subordonnée relative est inscrit dans l'appareil d'analyse institutionnel, que AM a sollicité pour rappeler un savoir ancien : la structure de la phrase de base, modèle auquel toute phrase est censée pouvoir se rapporter. Ensuite, AM a présenté une sorte de matérialisation de la formation des relatives, un mécanisme en deux mouvements, constituant sa propre transposition du modèle institutionnel : on va chercher l'antécédent d'un côté; on cherche le rôle (AM ne parle jamais de fonction) dans la deuxième partie de la phrase. En parlant de deuxième partie (ou de l'autre partie de la phrase), AM escamote l'élément subordonnée enchâssée dans la phrase de rang supérieur, jamais vraiment actualisé. Dans l'extrait en question, AM attire l'attention sur la variation des formes du relatif en 
fonction du rôle syntaxique et demande le retour réflexif sur le choix du pronom relatif ainsi que la verbalisation des critères adoptés. Les élèves s'engagent dans la réflexion sur ce qu'ils ont fait (des interventions sur des paires de phrases comportant un terme identique), mais ils ne se réfèrent ni à la structure de la phrase modèle, réactivée avec leur contribution et évoquée rapidement comme si elle était escomptée, ni au mode d'emploi de la relative proposé par AM. Ils s'écartent ainsi de la dimension de l'exemple, choisi pour montrer et valider un élément dans un système d'analyse connu, et ils tentent de trouver des réponses en s'appuyant sur la linéarité des unités et en créant des relations entre la forme du pronom et la signification de son antécédent : où remplace un lieu, que un objet ou une personne. Il s'agit de repères intuitifs, sans référence explicite à un savoir partagé, que AM conteste à partir des deux exemples : 1. J'aime Paris où j'ai passé mes vacances. 2. Je passe mes vacances à Paris que j'aime bien.

AM: hum hum (rires)// alors ICI:(montre successivement les énoncés) OÙ a remplacé un lieu/ d'accord et QUE a remplacé un lieu/ zut

Dans la dernière partie de l'enchaînement, AM reconstruit la relation entre la forme et la fonction du relatif avec le concours d'un élève. Pour le faire, il s'appuie sur sa procédure en deux mouvements.

Contrairement à AM, BS reconstruit l'objet selon les étapes du savoir institutionnel s'appuyant systématiquement sur le manuel. Faisant appel à des savoirs censés être disponibles, BS situe d'abord la relative parmi les subordonnées, ensuite parmi les compléments du nom. Le modèle de la phrase de base est également mobilisé pour représenter l'enchâssement et le déplacement en tête de phrase du constituant pronominalisé.

$\mathrm{Au}$ cours des deuxième et troisième leçons, les mêmes formes d'intervention sont répétées pour les relatives en qui, que dans la leçon 2, en dont dans la leçon 3. Il s'agit de retrouver la phrase de base correspondant à la subordonnée relative et de l'inscrire dans le schéma en arbre, ce qui devrait permettre d'identifier le constituant pronominalisé et surtout son rôle syntaxique. BS suit de très près le manuel et, dans le troisième enchaînement de la leçon 2, il s'y réfère pour reconstruire les traits pertinents de l'action : d'abord retrouver la phrase de base (phrase P) sous-jacente à la relative, ensuite rendre compte par une flèche du double rôle du relatif, substitut et outil d'enchâssement. La notion de transformation est ainsi matérialisée à l'aide du schéma de la phrase de base. Dans l'épistémologie de l'enseignant, ce schéma constitue l'explication et en même temps l'outil dont les élèves devraient se servir pour identifier la fonction du relatif.

Au cours du troisième enchaînement de la troisième leçon, il s'agit d'analyser le segment les arbres dont le forestier s'occupe. Bien que nos informations sur l'action des élèves soient partielles, on peut inférer de leurs questions que tout au moins ceux qui les posent segmentent leur action suivant les étapes proposées. Ces questions constituent en effet des interrogations sur l'application du modèle d'analyse, ce qui semble prouver qu'ils l'ont identifié comme procédure reproductible et qu'ils réagissent lorsqu'ils se heurtent aux limites de cette reproduction prétendument à l'identique. Ainsi, l'élève qui soulève la question sur la préposition, s'interroge sur la procédure de retour à la phrase $\mathrm{P}$, puisqu'il constate que le schéma simplifié ne peut être appliqué à l'identique pour les deux syntagmes :

les arbres que le bûcheron aime (le bûcheron aime les arbres)

les arbres dont le forestier s'occupe (le forestier s'occupe des arbres)

Elève: mais heu monsieur

BS: oui alors vas-y

Elève : mais pourquoi dans l'exercice avant le / on a mis

heu crochets on a mis les arbres on n'a pas mis des arbres

l'exercice précédent là

BS: l'exercice précédent

Elève : les quatre phrases

BS: heu : ah ici / alors les arbres / le bûcheron aime

les arbres là y a pas de préposition t'es d'accord

Elève : ah oueh oueh

BS: y a pas de préposition donc y a pas de raison de le 
modifier // d'accord / par contre ici / c'est c'est les arbres / c'est DE les arbres / alors pour faire DE les arbres et ben on contracte la préposition de et l'article heu enfin le la le déterminant défini les ça donne des / d'accord

Elève : oueh

L'élève soulève le problème de la complémentation et partant de la relation entre syntaxe et lexique, mettant ainsi en évidence que le traitement du lexique n'est pas intégré dans le schéma simplifié dont il dispose. En effet, le corpus d'exemples soumis aux élèves est construit en tant qu'instance d'observation et de validation de propriétés particulières. Dans quelle mesure l'enseignant adhère-t-il à cette construction préalable ? Et, corollairement, comment évalue-t-il l'écart éventuel entre les connaissances des élèves qui agissent et celles qui sont sous-jacentes à la construction des exemples ?

La difficulté du traitement des exemples se manifeste dans les deux séquences. Dans l'épistémologie de la théorie générative de référence, du moins idéalement, l'exemple fonctionne comme instance de validation d'une hypothèse. Dans les pratiques en classe, le statut de l'exemple se modifie, soumis au processus de la transposition et de la reconstruction institutionnelle. Il ne faut pas oublier, en effet, que l'exemple existe dans toute grammaire et qu'il y joue des rôles différents. Il oscille entre les données empiriques et les faits, considérés comme un fragment de théorie, une configuration minimale des données se prêtant à être théorisées. Dans son analyse du recours aux exemples comme constitution des faits et comme validation des données dans la tradition grammaticale française, Fournier (2007) montre que tous les exemples ne sont pas des données brutes « de la langue empirique représentée dans le discours grammatical » (p. 87). Ils peuvent être également des fragments de théorie schématisés.

Dans les pratiques observées, l'environnement mis en place ne révèle pas la construction de la distinction entre faits et données empiriques. Il laisse plutôt entrevoir un continuum, ou une sorte de transition naturelle qui se produirait notamment lors du passage à la production et à la compréhension écrite. Si cette attitude est confirmée par des analyses ultérieures, elle tend à montrer que le travail à partir de l'exemple, omniprésent dans les pratiques, n'est pas véritablement construit sur le plan didactique. En particulier, les interventions sur les données de langue, qui devraient aboutir à la mise en place de procédures codifiées, semblent ne pas être situées par rapport au double clivage mentionné: la distinction entre données brutes et fragments de schémas théoriques; la distinction entre les exemples à observer et à manipuler pour se forger une représentation de la langue et les exemples à retenir pour apprendre à produire et à comprendre selon les normes valorisées.

A partir de notre interprétation du sens de l'objet et des actions, nous nous interrogeons sur la rationalité de l'objet institutionnalisé, des formes d'actions relatives à cet objet et des interventions verbales et praxéologiques des élèves qui prennent la parole ou agissent de manière visible dans les données. Leur engagement dans l'action, parfois au-delà des attentes des enseignants, semble prouver que des objets inadéquats peuvent enclencher des formes d'action qu'il serait réducteur de ramener à une simple exécution laborieuse, sous l'autorité de l'enseignant. D'autres recherches seraient nécessaires pour situer les interventions des élèves par rapport à la rationalité des pratiques et des objets institutionnels ainsi que pour comprendre le développement de l'élève, en interaction avec le collectif de la classe et les conduites des enseignants.

\section{La grammaire en FLE : quel appareil d'analyse?}

La didactique du FLE, confrontée au problème de l'enseignement de la grammaire ou, plus largement, de la structuration de la langue au sens d'une grammaire élargie (Chartrand et Boivin, 2005) hésite à prendre position. Les réponses de la recherche sont assez fragmentaires. Par ailleurs, les enseignants de FLE reçoivent une formation qui comporte très souvent une approche de la langue issue de la tradition grammaticale. De même, dans les grammaires étiquetées FLE, les angles d'attaque présupposent tous une connaissance partagée de l'analyse grammaticale traditionnelle. Qu'en est-il des pratiques ? Est-il 
possible de les analyser dans la même perspective que les pratiques d'enseignement de la grammaire en FLP ?

\subsection{D'une didactique à l'autre}

L'enseignement du FLE est, par définition, destiné à des publics ayant leur propre tradition d'analyse d'une ou de plusieurs langues. On s'attend à ce que les pratiques d'enseignement de la grammaire soient extrêmement diversifiées, très éloignées de la conventionnalisation que l'enseignement obligatoire contribue à créer dans les pays francophones. Ensuite, on sait que dans l'enseignement du FLE et des langues en général, la langue cible constitue l'objet enseigné en même temps que le moyen pour l'enseigner, d'où l'effet de continuum susceptible d'occulter des objets particuliers. Enfin, les contenus à enseigner sont formulés en termes de compétences et de thèmes socio-culturels et ils sont évalués au travers de tâches communicatives, ce qui focalise ultérieurement l'attention sur le continuum langagier. Dès lors, est-il possible de décrire et comprendre ce qui se passe dans une classe de FLE en se demandant comment se construit l'objet enseigné, entité certes difficile à définir, mais correspondant incontestablement aux représentations sociales de l'enseignant, amené à rendre compte de ce qu'il a fait dans des textes institutionnels écrits ou oraux, ainsi qu'à celles des élèves, censés continuer, approfondir, réviser par un travail personnel ce qu'ils ont fait en classe ? Pour évaluer la pertinence de ces questions dans la phase exploratoire actuelle, nous avons commencé par tester les outils méthodologiques mis au point dans la recherche du GRAFE mentionnés précédemment. Nous sommes partie de deux principes: les formes que prend la reconstruction de l'objet dans la situation de classe sont d'abord déterminées par le travail enseignant et ensuite négociées dans le flux de l'action. Plus en amont, elles relèvent des conventions en vigueur dans les pratiques de l'institution d'enseignement.

Nous avons admis en effet qu'il existe une institution, l'enseignement du FLE à des publics non francophones ${ }^{5}$ en France, susceptible de favoriser l'émergence d'une certaine régularité des conduites et des objets. Les observations que nous avons effectuées dans le Centre Universitaire d'Etudes Françaises (CUEF) de l'Université Stendhal-Grenoble 3, institution créée il y a environ un siècle pour l'enseignement du français à des publics non francophones, en grande majorité étudiants, nous ont confirmé qu'il était possible d'identifier des régularités dans les pratiques de l'enseignement du FLE à des adultes scolarisés suivant une formation en France. Pour cerner ces régularités, nous avons utilisé des outils forgés pour l'analyse des pratiques en FLP. Alors que dans le projet en FLP évoqué supra, le but de la recherche était d'identifier les caractéristiques d'un objet spécifique, l'analyse des données de FLE vise à identifier, dans le flux des actions verbales et non verbales, des formes imputables à la mise en œuvre d'objets grammaticaux identifiés comme tels en situation. Il ne s'agit donc pas, dans la phase actuelle de notre recherche de comparer des données de FLP d'une part, de FLE d'autre part, mais plutôt de tester une double hypothèse : les objets enseignés sont de bons indices pour analyser ce qui se passe dans une classe ; les objets grammaticaux enseignés dans des classes de FLE sont construits selon des procédures identifiables.

\subsection{Des objets grammaticaux et des actions spécifiques ?}

Les données recueillies constituent une masse importante d'heures d'enregistrement et de pages de transcription $^{6}$. A la suite de plusieurs entretiens avec les enseignants qui se sont engagés à accueillir les observateurs, il s'est avéré très difficile d'envisager un contrat de recherche les engageant à mettre en œuvre un objet grammatical particulier, sans modifier leur propre planification. En effet, dans leur démarche, le traitement d'un objet donné s'étale sur plusieurs jours pendant un temps très limité, ce qui impliquerait des entrées et sorties d'observateurs pouvant perturber le déroulement du cours. Les contraintes institutionnelles n'ont pu être surmontées pour l'instant. Nos séquences sont donc hétérogènes $\mathrm{du}$ point de vue des objets, mais nous faisons l'hypothèse qu'elles donnent à voir les modalités d'alternance de ceux-ci dans l'organisation des cours intensifs, dans lesquels l'enseignant et les élèves sont soumis à l'impératif institutionnel de faire circuler la parole le plus possible. 
Les séquences enregistrées, une dizaine de quatre à six heures chacune, ont été synthétisées à l'aide d'un outil méthodologique, le synopsis, qui en fait une description narrativisée et hiérarchisée (Schneuwly, Dolz \& Ronveaux, 2006) et en donne une vue d'ensemble. Dans la phase exploratoire actuelle, seules quelques caractéristiques générales peuvent être dégagées. D'abord, l'alternance d'objets grammaticaux et d'objets textuels, relevant massivement de l'oral conversationnel, peu formalisé. Ensuite, l'organisation thématique des séquences : les données montrent que souvent les objets et les actions s'organisent autour du thème retenu. Une fois que la présence des objets grammaticaux est attestée, il s'agit de s'interroger sur leur sens en situation. Parmi les objets mis en œuvre, les verbes occupent une place importante : temps du passé, voix passive, participe, morphologie verbale... Nous commenterons brièvement ici deux séquences dans lesquelles un objet éminemment complexe, l'alternance passé composé/imparfait, joue le rôle d'organisateur de la séquence à égalité avec des objets textuels. Les deux séquences ont été filmées dans deux classes composées d'une quinzaine d'étudiants non francophones, de niveau intermédiaire, issus de langues et de cultures différentes.

Dans la séquence LM, nous avons retenu un extrait présentant la reconstruction d'exercices effectués au laboratoire. Dans une série de phrases, les apprenants ont complété les lacunes avec un verbe au passé composé, ensuite, revenus dans la salle de classe, ils doivent écrire la correction de l'exercice du laboratoire au tableau. L'enseignante prend la correction en charge et, après les phrases, il invite les apprenants à aborder une série de brefs récits. Comme dans les phrases, l'opposition est justifiée par la valeur d'aspect ponctuel des verbes au passé composé et d'aspect duratif des verbes à l'imparfait. Les verbes des phrases ont en effet été sélectionnés pour permettre ce type de justification, qui relève de l'épistémologie de l'enseignant et de l'élémentarisation de l'objet dans la séquence observée. Mais au cours des échanges, émerge également un élément relevant de la textualisation comme le montre ce fragment d'institutionnalisation (minute 21).

\begin{abstract}
E : pourquoi on utilise ça / et pourquoi on utilise ça $\uparrow / /$ ici / c'est les circonstances les circonstances / samedi / la pluie euh le monde / beaucoup de monde / tous les vendeurs occupés (...) ça c'est la situation / et ici pam pam pam pam pam pam j'ai fait ça j'ai fait ça j'ai fait ça j'ai fait ça j'ai fait ça j'ai fait ça j'ai fait ça / ici la situation / Carrefour samedi après-midi extérieur / pluie / intérieur / du monde du monde du monde du monde les vendeurs occupés / situation alors moi $\uparrow / \mathrm{j}$ 'ai fait ci j'ai fait ci j'ai fait ci //
\end{abstract}

On peut cerner, dans certaines des actions, les régularités des pratiques grammaticales traditionnelles du travail sur les exemples, mais d'autres pratiques sociales sont sollicitées en permanence, en particulier la simulation de situations d'interaction dans des lieux sociaux divers. Ceci demande la disponibilité du lexique, donc la définition et la paraphrase interviennent régulièrement. Les difficultés d'intercompréhension dans des groupes plurilingues suscitent également la dimension phonétique. Dans la séquence LM, celle-ci s'installe progressivement jusqu'à se combiner avec l'objet grammatical : pour les apprenants, il s'agit d'observer et de répéter des exemples pour identifier la forme des verbes mais également pour produire l'intonation attendue. L'intervention ne se fait plus à tour de rôle, mais tout le groupe répète. La pratique de l'enseignement de la grammaire fait place à une pratique autre, proche de la récitation et du théâtre, et il devient difficile d'identifier quelle est la dimension dominante de l'objet.

Dans la séquence LR, on retrouve un élément de l'objet grammatical analogue à celui identifié dans la séquence LM : l'opposition passé composé/imparfait est assimilée à l'opposition de la durée des actions dans des situations de la vie courante, comme le montre l'extrait ci-dessous. En revanche, l'opposition entre situation et événement, selon les étiquettes terminologiques de LR, n'est pas actualisée dans la dimension de la textualisation. Soulignons toutefois que la séquence enregistrée ne fournit qu'un aperçu de l'objet et des conduites de l'enseignant.

E : Alors je voudrais rappeler pour ceux qui ont quelques difficultés la différence du choix entre la passé composé et l'imparfait hein le passé composé c'est un événement heu / un événement passé et l'imparfait est une situation hein donc vous voyez on va reprendre l'exemple qui était / qui est le premier ici / quand ma mère a retiré la gâteau du four quand ma mère a retiré le gâteau du four tout le monde comprend four (les apprenants montrent q’ils ont compris) le gâteau était en en train de brûler ou il a 


\begin{abstract}
brûlé vous voyez il a brûlé c'est pas possible quand ma mère a retiré le gâteau du four vroum il a brûlé ( $E$ fait le geste de tirer un objet vers elle et ensuite elle lève les mains vers le haut) c'est pas possible c'est situation il était en train de tout le monde comprend être en train de d'accord
\end{abstract}

De cette première phase exploratoire, émerge qu'en FLE comme en FLP, l'enseignant conçoit sa leçon en termes d'objets et d'actions reliés entre eux, ce qui n'est sans doute pas surprenant. Les séquences enregistrées révèlent l'alternance d'activités de classe autour d'objets grammaticaux - souvent en préparation ou en rappel du travail fait au laboratoire de langue - et d'objets discursifs. La succession de ces objets est très souvent annoncée en début de séquence par l'enseignant. Enfin, non seulement les objets grammaticaux sont présents, mais ils structurent la séquence au niveau le plus élevé.

Reprenant la discussion sur l'exemple, je dirai que dans les données actuellement disponibles, la grammaire pour parler correctement domine, renforcée par la mise en situation de l'exemple; la grammaire pour décrire, pour réfléchir sur les régularités de la langue est minoritaire et semble relever du travail personnel de l'apprenant. Du point de vue des dimensions du langage, les dimensions de l'analyse et de l'usage situé sont très imbriquées dans la construction de l'objet en classe. Cela entraîne des déplacements très rapprochés du rôle des interactants et des pratiques langagières. La pratique de l'exercice - chacun lit une phrase et la manipule pour produire la forme attendue - est souvent connue par les élèves, elle est répétitive et comporte pour eux des risques raisonnables. Au cours de cette activité de classe, l'enseignant introduit de manière impromptue une nouvelle pratique, engageant des échanges sur le modèle de la conversation amicale. Les contraintes changent : la consigne ne consiste plus à apporter une modification à l'exemple mais à essayer de mobiliser sa connaissance de la langue pour s'exprimer de manière adaptée aux conventions établies dans le groupe. Selon le contrat didactique intériorisé et les habitudes culturelles des apprenants, cela comporte des difficultés plus ou moins importantes.

Au cours des activités observées, les dimensions du langage sont imbriquées selon des conventions très largement implicites. La recomposition des dimensions qui aurait pu se mettre en place à partir de l'analyse grammaticale illustrée dans le Niveau Seuil, fondée sur les travaux des grands sémanticiens français comme Guillaume et Pottier, n'a pas véritablement abouti. Parallèlement, la dimension du texte ${ }^{7}$ est aujourd'hui théorisée dans des disciplines diverses et l'analyse des interactions, approche théorique dominante en DFLE, n'est pas encore parvenue à fédérer ces points de vue multiples. Au niveau des pratiques, la recomposition des dimensions se fait de manière empirique et encore mal connue. L'analyse des objets enseignés selon l'approche méthodologique adoptée, par l'explicitation du parcours transpositif ainsi que des reconstructions internes à l'institution, devrait permettre d'identifier des critères pour rationaliser les interventions sur le terrain. En particulier, les résultats de ce type de travaux pourraient aider à comprendre en quoi les mécanismes de l'approche communicative dominante peuvent être améliorés, notamment avec des publics issus de cultures différentes et parfois très éloignées, ne possédant ni les conventions sociales implicites de la conversation ordinaire en français ni le contrat didactique impliquant les glissements systématiques d'une dimension à l'autre.

\title{
4 Eléments de conclusion
}

Ce regard transversal sur les objets grammaticaux enseignés en FLP et en FLE fait apparaitre des régularités analysables. Les représentations des objets et des activités grammaticales en classe font souvent référence à la logique construite dans les documents présentant le contenu apprêté pour être enseigné. Or les objets reconstruits au travers de l'analyse critique des pratiques sont forcément en décalage par rapport aux représentations de ce qu'ils devraient être et de la manière dont ils devraient fonctionner. Cette disjonction apparaît comme plus ou moins problématique pour l'institution d'enseignement et plus largement pour la société. En FLP, les objets grammaticaux enseignés ont une visibilité sociale plus forte et sont exposés à des évaluations nettement plus généralisées qu'en FLE. Cet entour socio-politique contribue sans doute à renforcer la distinction entre les deux didactiques de la grammaire. En attendant que l'analyse des pratiques nous aide à cerner les convergences et les divergences des deux domaines d'enseignement et d'apprentissage, le discours idéologique sur les objets 
qui doivent être enseignés et sur les conduites qui doivent en faciliter l'apprentissage risque d'occuper une place prédominante.

Quant aux actions, dans l'appareil d'analyse de FLP évoqué, elles sont préfigurées à l'aide de la notion de manipulation. Dans notre étude de cas, les manipulations en situation sont interprétables comme une procédure mécanique de classement, plutôt que comme un test. La valeur de test de l'opération syntaxique est gérée totalement par l'enseignant, qui aménage l'environnement afin d'éliminer les alternatives. Lorsque des variables sont introduites par les élèves, les enseignants ne les prennent généralement pas en compte, sans doute pour ne pas s'écarter de l'objectif visé. Les élèves ne font pas la distinction entre donnée empirique et exemple comme instance de validation d'une hypothèse, donc comme entité déjà construite pour l'expérimentation. Le décalage entre la recherche théorique qui est à l'origine des manipulations et les manipulations en classe est inévitable. Une des questions qui relèvent de la didactique est dès lors la suivante : le sens des manipulations observables justifie-t-il la pertinence de l'objet par rapport à la rationalité globale de l'appareil d'analyse ? Cela nous ramène à un débat bien connu que nous n'avons pas la prétention de trancher: comment faire mieux quant on enseigne la grammaire en classe. Faut-il renoncer au principe de la validation des propriétés à l'aide de manipulations qui fonctionneraient comme test? Cela renforcerait la grammaire pour bien écrire et le recours à l'exemple comme modèle d'une langue normée, que l'école se doit de toute façon d'enseigner. Inversement, l'emploi de procédures que les élèves seraient capables de gérer, renforcerait l'étude de la grammaire comme ensemble de principes explicites, aptes à rendre compte d'une ou de plusieurs dimensions du langage. Ce choix comporterait la capacité à analyser les énoncés que les élèves produisent ou une sélection de ceux-ci, effectuée selon des critères explicites et partagés.

En FLE, le sens des objets grammaticaux et des procédures adoptées dans les pratiques est encore très mal connu. Nos premiers résultats tendent à confirmer la présence de reconstructions d'objets selon des conventions qu'il serait utile de mieux décrire et comprendre. Apportant des éclairages nouveaux, l'analyse critique des pratiques permettrait de préciser les questions de recherche sur le contenu, son enseignement et son apprentissage. Cela contribuerait à construire des positionnements théoriques sur la base desquels la didactique serait mieux à même de mettre en synergie les descriptions et les modélisations multiples du langage et de ses dimensions, nécessaires à la définition des objets à enseigner. Cette phase de la recherche serait ensuite prolongée par la technologie d'intervention sur le terrain, le rôle social de la discipline étant de construire des dispositifs adaptés afin que l'efficacité préconisée dans les discours en amont de l'enseignement se concrétise dans les pratiques en classe, au travers des objets mobilisés et des actions qui en définissent les contours.

\section{Bibliographie}

Béguelin, M.-J. (2000) (Ed.). De la phrase aux énoncés - Grammaire scolaire et descriptions linguistiques. Bruxelles : De Boeck - Duculot.

Besson, M.-J., Genoud, M.-R., Lipp, B. \& Nussbaum, R. (1979). Maîtrise du français. Lausanne : Office romand des éditions scolaires.

Bronckart, J.-P. (1996). Activité langagière, textes et discours - Pour un interactionnisme socio-discursif. Neuchâtel et Paris : Delachaux et Niestlé.

Bronckart, J.-P. (2004). Pourquoi et comment analyser l'agir verbal et non verbal en situation de travail ? In J.-P. Bronckart \& groupe LAF (Ed.), Agir et discours et situation de travail (pp. 11-144). Université de Genève : Cahiers de la section des sciences de l'éducation, 103.

Brousseau. G. (1998). Théorie des situations didactiques. Grenoble : La pensée sauvage.

Canelas-Trevisi (1997). La transposition didactique dans les documents pédagogiques et dans les interactions en classe. Thèse de doctorat non publiée en sciences de l'éducation, Université de Genève.

Canelas-Trevisi (sous presse). La grammaire enseignée en classe. Le sens des objets et des manipulations. Berne : Peter Lang. 
Chartrand, S.-G. \& Paret, M.-C. (1995). Langues maternelle, étrangère, seconde : une didactique unifiée ? In J.-L. Chiss, J. David \& Y. Reuter (Ed.), Didactique du français, état d'une discipline (pp. 197-208). Paris : Nathan.

Chartrand \& al. (2003). Le programme de français du secondaire : état des lieux et perspectives, Québec français, 128.

Chartrand, S.-G. \& Boivin, M.-C. (2005). Articulation des activités métalinguistiques aux activités discursives dans la classe de français au secondaire inférieur. In E. Falardeau (Ed.), Le français : discipline singulière, plurielle ou transversale? Actes du $9^{e}$ colloque international de l'AIRDF [CD-ROM]. Québec: AIRDF.

Fournier, J.-M. (2007). Constitution des faits / validation des données dans les grammaires de la tradition française. Langages, 166, 86-99.

Milner, J.-C. (1989). Introduction à une science du langage. Paris : Seuil.

Schneuwly, B., Dolz, J. \& Ronveaux, Ch. (2006). Le synopsis : un outil pour analyser les objets enseignés. In M.J. Perrin-Glorian, \& Y. Reuter (Eds.), Les méthodes de recherche en didactique (pp. 175-190). Villeneuve d'Ascq : Presses Universitaires du Septentrion.

Schneuwly, B. \& Dolz, J. (en préparation). La construction de l'objet enseigné en français. Analyse du travail de l'enseignant en classe sur la grammaire et sur la production écrite.

Schubauer-Leoni, M.L., Leutenegger, F., Ligozat, F. \& Flückiger, A. (2007). Un modèle de l'action conjointe professseurs-élèves : les phénomènes didactiques qu'il peut/doit traiter. In G. Sensevy \& A. Mercier (Ed.), Agir ensemble. L'action didactique conjointe du professeur et des élèves (pp. 51-91). Rennes : Presses universitaires de Rennes.

Sensevy, G. (1998). Institutions didactiques. Etudes et autonomie à l'école élémentaire. Paris : PUF.

\footnotetext{
${ }^{1}$ Nous considérons que le classement des savoirs - le savoir savant, issu des pratiques de production du savoir scientifique, censé bénéficier d'une légitimation indiscutable, les savoirs d'expertise, de sens commun issus des pratiques sociales de référence, nettement plus fragiles - ne permet pas de construire des analyseurs pertinents. Cette répartition contribue à entretenir l'illusion qu'il est possible d'autonomiser des savoirs, en les isolant des genres de textes dans lesquels ils fonctionnent et des pratiques sociales où ces genres prennent tout leur sens. Or les savoirs constituent des biens cognitifs socialement et historiquement situés, ils se concrétisent dans des genres de textes selon des schémas d'exposition et en même temps de valorisation et ils sont soumis à une évaluation qui en fixe le degré de légitimité, susceptible de se modifier dans le temps. Il revient au didacticien la responsabilité épistémologique de construire une prise de distance explicite par rapport aux savoirs académiques des disciplines connexes ainsi qu'aux savoirs de toute pratique sociale retenue comme référence pour l'enseignement.

${ }^{2}$ Les formes d'action se construisent sous l'effet de la réflexivité dont témoignent les protagonistes, aussi bien les observateurs externes que les actants directement impliqués dans l'action (Bronckart, 2004, p. 113). Tout individu oriente son interprétation à partir des modèles déposés dans les représentations collectives et le chercheur s'inscrit dans ce processus interprétatif, qu'il reconstruit avec ses outils. L'individu puise dans les représentations collectives des critères pour interpréter et évaluer les activités et les actions ainsi que des modèles susceptibles d'orienter son interprétation.

${ }^{3}$ Le terme de finalité est employé ici en relation avec des indices matériels de construction institutionnelle de l'objet et n'implique nullement les représentations des enseignants et des élèves.

${ }^{4}$ Il s'agit de l'avant-dernière année pour la classe AM et de la dernière année pour la classe BS.

${ }^{5}$ Il est question ici de publics adultes ayant un niveau de formation équivalent au moins à l'entrée en licence. On peut faire l'hypothèse que les régularités qui se stabilisent en France pour ce type de public se maintiennent au moins en partie, dans les institutions françaises d'enseignement du français à l'étranger.

${ }^{6}$ Les conditions matérielles du recueil sont analogues à celles mentionnées précédemment pour les classes de FLP du secondaire.

${ }^{7}$ Le Cadre européen commun de référence pour les langues (2001) considère que les usages de la langue se concrétisent en une pluralité de textes « est défini comme texte toute séquence discursive (orale et / ou écrite) inscrite
} 
ISBN 978-2-7598-0358-3, Paris, 2008, Institut de Linguistique Française

Didactique et enseignement, français langue maternelle, français langue seconde DOI $10.1051 / \mathrm{cmlf0} 8298$

dans un domaine particulier et donnant lieu, comme objet ou comme visée, comme produit ou comme processus, à activité langagière au cours de la réalisation d'une tâche » (p. 15). Cette définition du texte, englobante, implique une conception du langage en situation. 